Chapter 14

\title{
Coronary Angiography - Technical Recommendations and Radiation Protection
}

\author{
Maria Anna Staniszewska \\ Additional information is available at the end of the chapter \\ http://dx.doi.org/10.5772/54034
}

\section{Introduction}

According to recent World Health Organization statistics for 2007, cardiovascular deaths account for $33.7 \%$ of all deaths worldwide, whereas cancer represents $29.5 \%$, other chronic diseases $26.5 \%$, injury and communicable diseases $4.3 \%$. [1]. Coronary artery disease (CAD) is the leading cause of cardiovascular death throughout the world. In the light of this, special attention has to be paid to CAD.

Imaging is an integral part of diagnostics and treatment of the disease, especially of surgical and transcatheter cardiological interventions. Pre-procedural imaging provides detailed understanding of the operative field prior to the procedure.

Traditional two-dimensional (2D) imaging, i.e. standard echocardiography and conventional angiography, relies on acquisition of a limited number of planes/projections, which cannot be changed during the review, while three-dimentional (3D) imaging allows fast acquisition of volumetric data sets and subsequent off-line reconstructions along unlimited 2D planes and 3D volumes. 3D imaging of the cardiovascular structures is pursued with computed tomography, magnetic resonance and also echocardiography.

Cardiovascular surgery is a dynamically developing field and many procedures are performed under control of dedicated imaging systems but most of these procedures start from coronary angiography.

Quantitative coronary angiography is the method for visualization of coronary vessels during exposure to x-rays, after their filling up with contrast agents. This allows to assess coronary artery stenoses for hybrid revascularization or quantifying stenoses in bypass grafts.

Depending on a further proceeding expected for a patient two versions of the procedure are possible: CCA - conventional coronary angiography and CTA- CT angiography. 
CCA is performed under the control of x-ray unit with C-arm and is preferred if further cardiovascular surgery is planned for the patient.

CTA is performed under the control of computerized tomography (CT scanner) and has only diagnostic meaning.

This chapter is divided into three parts concerning both: the techniques of coronary angiography (CCA and CTA) and the new technical solutions in this field. Some details concerning the standards of CCA and CTA performance have already been published [2] and are used here with addition of new information.

\section{CCA}

\subsection{Description of the procedure}

A patient lies in supine position on the table being a part of C-arm unit. X-ray tube moves rotationally in two perpendicular planes (horizontal and vertical) which makes any required type of projection possible. (The most common projections are: LAO, LAO/Cranial, LAO/ Caudal, RAO). The principal current-voltage parameters are chosen automatically.

Good practice rules require that the x-ray tube is kept under the table. This allows to avoid unnecessary irradiation of the staff and makes the doses to patient lower.

In order to visualize coronary vessels a contrast agent is administered intravenously: a thin catheter is previously introduced to the brachial artery or the femoral artery (there are two alternative access routes) and its movement is traced by fluoroscopy and observed on monitor by the operator.

(The physiological parameters of a patient are permanently monitored during the procedure.)

The operating team consists of 3 to 4 persons: usually they are an operator (with an assistant if necessary), a scrub nurse and an anaesthetic nurse. All the persons stay around the patient table and thus may be exposed to even high doses of radiation. (There are scattered x-rays mainly.) An especially exposed member of the team is the operator: the doses registered for such persons may achieve the highest level measured for occupational exposures [3,4].

Patient exposure results mainly from primary $x$-ray beam which covers a part of back surface (the area of left shoulder) by most time of the procedure. The remaining body of a patient is exposed to rather scattered radiation.

Three types of images can be created during the procedure:

a. Real-time images (fluoroscopic images) observed by the operator (visible also on the technical console),

b. Radiographic images for saving some chosen moments of the procedure (so-called acquisition), 
c. The long periods of exposure can be recorded in so-called "cine-mode" (if x-ray system is technically predicted to this mode of work).

The results of CCA procedure are analyzed by cardiologists during its performance and also retrospectively after that.

\subsection{Technical requirements for $\mathrm{x}$-ray systems with C-arm}

Cardiovascular surgery covers a wide array of procedures and methods, each with specific imaging requirements. Consequently, $x$-ray systems are offered by the vendors in many versions.

Two types of construction are proposed generally: a fixed C-arm system and mobile C-arm.

A fixed C-arm system can be floor-mounted or ceiling-mounted. The solution is chosen by the user in dependence on the space condition of operating room. Fixed C-arm systems are required for CCA and further surgery procedures such as: percutaneous coronary interventions (PCI) and endovascular aortic repair (EVAR).

A powerful mobile C-arm can be used for pacemakers or defibrillator implants and carotid artery stenting. However, fixed C-arm systems can offer added security and are preferred for complex cases and lengthier procedures.

Diagnostic procedures and a structural heart disease treatment in children using imaging system require a high temporal resolution, which is provided by dedicated fixed C-arm systems with very high frame rates.

Any x-ray system used for CCA procedures should be equipped as follows:

- high frequency converter generator of minimum $80 \mathrm{~kW}$ (in possibly rectangular pulses),

- $\mathrm{x}$-ray tube focuses not larger than $1.2 / 0.5 \mathrm{~mm}$,

- minimum two dose-rate modes (Low-Medium-High or Low-Standard),

- x-ray tube - image detector distance tracking (minimum focus skin distance $30 \mathrm{~cm}$ ),

- patient table made from low-attenuating materials,

- display of fluoroscopy time, total dose-area product (fluoroscopy and radiographic) and estimated skin entrance dose,

- image hold system (for example default storage of the last $20 \mathrm{sec}$. of fluoroscopy for reference or archiving).

Despite of the general features, the system should fulfil the following requirements:

- generator should be controlled by microprocessor, power switch accurately controlled, with short switching time (about $1 \mathrm{~ms}$ ); voltage should cover the range of (40-120) kV,

- a high power tube with high heat dissipation,

- dose-area product meter with display visible for operator, 
- should be equipped with additional filtration ( $\mathrm{Cu}$ preferred), enabling to select a proper filtration in dependence on x-ray spectrum,

- overcouch image detector,

- collimators incorporating circular shutters,

- flexibility of pulsed fluoroscopy mode (grid- switched and individually programmed with a different composition of x-ray dose rate, digital processing and filter settings),

- staff protective shielding,

- a possibility of AEC mode choice by the user (IMAGE or DOSE weighted).

Additionally in some x-ray systems are also available partially absorbent contoured filters (so-called wedge filters) to absorb radiation in the low density areas surrounding the heart.

Another proposal for coronary angiography appears in 2009 [5], i.e. biplane system in which simultaneous accurate images of the heart are provided from two different points of view. This allows to reduced contrast volume and radiation exposure. Biplane angiography helps to visualize complex coronary or structural cardiac anatomy. The clinical settings in which biplane system is the most critical occur in the paediatric population and in those patients with renal failure. The value of biplane angiography must be balanced against cost and difficulty of use: such system requires the patient's heart to be in the exact centre of the two planes (the isocentre) and then AP and lateral planes were properly angled in orthogonal protections.

\subsubsection{Image recording}

Most of x-ray units used in cardiology nowadays are equipped with the digital image recording system. There are two types of it: (1) an image intensifier based system and (2) a flat-panel fluoroscopy system. The first one utilizes conventional technology: the output screen of image intensifier is projected in a video camera or a CCD camera to produce an electronic information. Such systems are still used in many interventional labs. The older systems have got image intensifiers, which cause a significant loss of signal intensity because of the construction principle.

The digital Flat Detectors (FD) are smaller and easier to operate. Image quality may be better, but the modern $\mathrm{x}$-ray systems have much higher output capacity and thus higher doses are probable.

Maximum field of view FD has to be large enough: 30x36 cm (11.8x14.4 inch), but the used area may be part of the maximum in dependence on current medical needs.

Image matrix size should be also large - especially for the acquisitions recording, and can be smaller for fluoroscopic observations (the signals are then summarized).

Nyquist frequency of FD fluoroscopy systems are better than $3 \mathrm{lp} / \mathrm{mm}$.

Quite important are the monitors in the system: they can be monochrome or colour LCD of 18-19 inch. 


\subsection{Dosimetric quantities}

Presently used fluoroscopic equipment measures air kerma with the use of an ionization chamber incorporated into the x-ray tube envelope and reports the dose-area product (DAP), equal to air kerma (dose) multiplied by $x$-ray beam cross-sectional area. (This is legal requirement.) X-ray systems used for fluoroscopic procedures are obligatory equipped in DAP-meter to summarize emission in all the modes of work (i.e. fluoroscopy, radiography, cine). (Dose Area Product= DAP $\left.\left[G y \cdot m^{2}\right]\right)$. Measurements are performed by the transmission chamber placed on the $\mathrm{x}$-ray tube output.

$\mathrm{DAP}$ is independent on the distance from x-ray source ( according to the inverse-square law). Some systems show also peak skin dose (in grays) treated as the highest dose received by any location on the patient's skin, including both incident and back-scattered radiation. Although thought to be the best predictor of skin injury, peak skin dose is difficult to measure in practice and the values displayed by the x-ray system can be treated as an approximation of real value.

DAP (and entrance air kerma) values have to be displayed on the monitors of x-ray system and should be registered in the patient record. (Especially when entrance air kerma is over $1 G y$ and exceeds the threshold of deterministic skin effects.)

For a given projection mean entrance skin dose (or entrance air kerma) can be calculated as the ratio of DAP and the area of the incident x-ray beam (at the level of patient's skin). The relationship is not valid for DAP summarized for the whole procedure because of the incidence angle variation.

The actual dose received by a patient from a given x-ray procedure can be evaluated by the following approaches:

a. with calculation based on dosimetric measurements made in physical phantoms,

b. with Monte Carlo simulations using mathematical phantoms,

c. with DLP values provided by the $x$-ray system and a body region-specific conversion coefficient averaged for multiple scanners.

Dosimetric measurements are commonly made with termoluminescent dosimeters (lithium fluoride or calcium fluoride) or solid state detectors. The detectors are distributed inside the phantom which is exposed as a patient. The most popular are Rando Man or Rando Woman phantoms (representing adult humans) and the family of CIRS phantoms (representing children as well as adults).

Monte Carlo simulation assumes a mathematical phantom representing a patient from a given age group and model photon transitions through this phantom. The older solutions used the "family" of Cristy' phantoms for simulation, modelling organs as geometrical solids. The newer studies use voxel phantoms which are more anatomical being created on the basis of CT human images.

The last approach, i.e. taking some conversion coefficients from literature and multiplying by DAP is the simplest but on the condition that the values are really adequate for the given 
procedure and type of $x$-ray system. The range of conversion factor reported for CCA varies from 0.12 to $0.26 \mathrm{mSv} /\left(\mathrm{Gy} \cdot \mathrm{cm}^{2}\right)[6]$.

Regardless of the way, the effective dose estimates the radiation charge for some "typical" (standard) patient, without taking into consideration the anatomy of a given human, and thus should be referred to the category of patients undergoing the given x-ray procedure.

\subsection{Doses to patients}

The doses obtained by the patients undergoing CCA procedures depend on:

- patient architecture (BMI-body mass index),

- emission effectiveness of the x-ray system,

- applied dose-rate mode,

- total exposure time,

- mode of work (number of acquisitions -radiographic or cine).

Doses received by patients in CCA are evaluated both for the whole body and to the skin.

The entrance surface dose to patient dramatically increases when the focus-to-skin distance becomes too short.

The patient doses are significantly higher when the high dose-rate mode is activated or if pulsed fluoroscopy of high number of pulses per second is chosen. These doses are also inversely proportional to the size of image detector.

During coronarography an entrance dose for a small area of patient's back can be very high while a dose to the remaining part of the trunk can be very low. In consequence, the effective dose may be low and the deterministic effects can appear. (Skin injuries for patients undergoing interventional procedures are reported [7].)

\begin{tabular}{llll}
\hline Number of patients & \multicolumn{1}{c}{ DAP $\left[\mathrm{Gy. \textrm {cm } ^ { 2 } ]}\right.$} & \multicolumn{1}{c}{ Time of fluoroscopy $[\mathrm{min}]$} & Number of frames \\
\hline 130 & $72+/-55$ & $0.35+/-0.25$ & $1550+/-775$ \\
\hline 78 & 73 & 9.9 & 1079 \\
\hline 100 & 60.6 & - & 412 \\
\hline 117 & $1.1-11.3$ & $0.3-22$ & $39+/-11$ \\
\hline 106 & $35-160$ & $4.8+/-3.5$ & $30+/-10$ \\
\hline 62 & $37-190$ & $4.2+/-3.0$ & 639 \\
\hline 90 & $3.1-57.2$ & $1.5-5.1$ & $200-1911$ \\
\hline 194 & $64-281$ & $0.4-33$ & \\
\hline
\end{tabular}

Table 1. The doses to patients in CCA procedures (according to [8]) 
Paediatric patients also undergone CCA procedures. The doses for 50 children examined using Allura Xper F20 system are given in Table 2 (the own data from the paediatric clinic).

\begin{tabular}{cc}
\hline Number of patients & The range of PSD [mGy] $\left(^{*}\right)$ \\
\hline 6 & $0-20$ \\
\hline 7 & $20-40$ \\
\hline 2 & $40-60$ \\
\hline 7 & $60-80$ \\
\hline 5 & $80-100$ \\
\hline 11 & $100-200$ \\
\hline 4 & $200-300$ \\
\hline 4 & $300-400$ \\
\hline 3 & $400-500$ \\
\hline 1 & $" />500$
\end{tabular}

${ }^{*}$ ) the value of Peak Skin Dose is displayed by $x$-ray system

Table 2. The values of PSD [mGy] for paediatric patients during CCA

The values of effective doses in non-paediatric population undergoing CCA is presented and discussed in [6]. The values were published during ten years (from 1997 to 2007). The mean value is $7.4 \mathrm{mSv}$ but standard deviation is very high $(5.4 \mathrm{mSv})$ because of a wide range of reported doses (from 2.3 to $22.7 \mathrm{mSv}$ ). Similar value of $\approx 7 \mathrm{mSv}$ as typical effective dose for adult patients in CCA is also cited by the UNSCEAR [6].

\subsection{Doses to staff}

Radiation risk to staff in CCA procedures is caused by necessity to work in the radiation filed, standing near patient during exposure. Staff is exposed mainly to scattered radiation although accidentally can be irradiated by primary $\mathrm{x}$-rays.

The factors affecting staff dose are as follows:

- relative position with respect to patient,

- patient architecture (BMI),

- irradiated patient volume,

- $x$-ray tube position,

- time of exposure,

- effective use of protection shielding. 
Thus, staff and patient doses are partially linked: higher exposure for a patient means higher irradiation to staff. This is especially true for the operator and the person standing nearby him (an assistant or scrub nurse). Additional factor determining the doses to staff is professional experience and good training in the procedure performance: good manual skills cause the exposure time shorter.

The problem of radiation risk to staff in interventional cardiology had been widely discussed many times.The essential conclusions resulted from that are the recommendations concerning performance of the interventional procedures and elaboration of the methodology for evaluation radiation risk for the staff $[4,9]$.

According results of the survey performed by European research group SENTINEL in a sample of European cardiac centres [10] doses for the first operator are as follows:

a. annual effective dose: median $1.3 \mathrm{mSv}$, third quartile $1.4 \mathrm{mSv}$,

b. equivalent dose over the apron: median $11.1 \mathrm{mSv}$, third quartile $14 \mathrm{mSv}$.

Although the above values are rather low it should be underlined that these values may be higher many times (even up the annual limits), especially for inexperienced staff or in a case of clinically difficult patient.

Currently Philips as the only global healthcare company offered a real-time dosimetric system Dose Aware. The system does not replace the thermoluminescence dosimeter (TLD) as a legal dose meter. TLD reports accumulated x-ray dose from the exposure for a period of time but does not include any time stamp or awareness where and when the x-ray dose was acquired.

Dose Aware is a system that gives real-time feedback of scattered x-ray dose reception and instant access to time-stamped dose history. The system is completed from:

- Base Station : an LCD touchscreen displays real time dose data from all PDMs within range. The Base Station stores PDM data as well. Multiple Base Stations can be network linked to a computer running Dose Manager software for analysis.

- Personal Dose Meter (PDM) -worn by the staff; smart badge measures scatter radiation and transmit this information to the Base Station where is displayed.

- Cradle: for placement of the PDM (outside of the exposure time)

- Dose View: PC software package is included together with the Base Station.

Staff working in an x-ray environment wears a PDM. This PDM measures x-ray dose reception and is wirelessly connected to the Base Station. The Base Station is mounted in the examination room where all staff can directly see whether received dose is in red, yellow or green area. The "yellow" or "red" status means a necessity of immediate action to reduce x-ray exposure. X-ray dose history information can be automatically retrieved from any Base Station or from any PDM by using a Cradle with Dose View software or Dose Manager software. 


\section{CTA}

Cardiac CT procedures were an engine for development of new technical solutions in CT: visualization of quickly moving anatomical structures needs extremely short time for acquisition and reconstruction of images. This is available in the new multislice CT scanners, with rotation time not longer than $0.4 \mathrm{~s}$ and slice collimation $8 \mathrm{~cm}$ or more (up to $16 \mathrm{~cm}$ ). Improvements in spatial and temporal resolution, scan time, scan range and advanced image postprocessing (very important in clinical practice) have made CT angiography (CTA) an excellent tool for identifying patients in need of invasive therapy and for mapping out the best percutaneous or surgical approach. In some cases CTA provides complementary information to that of conventional angiography (CCA).

Although history of cardiac CT began with the four-slice scanners, the real development started with 64-slice scanners with appropriate combination of spatial and temporal resolution. However, the intensity of x-rays emitted in the multislice CT scanners has to be higher and then a number of dose-reduction mechanisms is implemented for patients' safety.

According the American College of Cardiology Foundation (ACCF) the principal indications to cardiac CT are as follows [11]:

- low and intermediate pretest probability of obstructive coronary artery disease (CAD),

- noncontrast CT calcium scoring for patients at intermediate risk of coronary heart disease and for low-risk patients with a family history of disease,

- coronary CT is especially recommended for patients with reduced left ventricular ejection fraction at low or intermediate pretest probability of disease,

- preoperative CT angiography for both heart surgery and noncoronary indications in the setting of risk of CAD.

The current role of coronary CT angiography in the assessment of coronary artery disease is based on its high negative predictive value in ruling out significant stenosis in intermediate risk populations. [12]. A precise preprocedural visualization of complex coronary lesions with CT angiography would be complementary to conventional angiography and allow optimization of the interventional approach during subsequent PCI [13].

The main benefit from CTA is evaluation of cardiac structure and function and also evaluation of anatomical structures surrounding the heart.

\subsection{Description of the procedure}

From technological reasons the possibilities of $\mathrm{CT}$ are limited to diagnostics, without further therapeutic activity (means angioplasty).

The main stages of CT study protocol are as follows:

1. the heart rate control and regulation,

2. calcium scoring, 
3. i.v. injection of contrast agent,

4. CT angiography.

As coronary disease is jointed with calcification of coronary arteries, evaluation of this process is a good indicator of pathology. CTA gives possibility to display calcifications (3D) and to assess them quantitatively: it is so-called "calcium scoring".

Generally, the heart can be imaged well if its structures do not move significantly during the scanning time. This condition is fulfilled during the diastolic phase of cardiac cycle. For selection of the proper phase for CT images recording ECG gating (triggering) is applied.

General rules for exposure parameters:

Slice thickness $<1 \mathrm{~mm}$, pitch $<1, \mathrm{t}_{\mathrm{rot}} \leq 0.5 \mathrm{sec}$, kilovoltage $\mathrm{U} \geq 100 \mathrm{kV}$, mAs and physical filtration are dependent on the CT scanner, ECG-gating.

Set of parameters for a given CT scanner is determined by the model of a scanner and the version of software dedicated to coronary procedures and offered by the vendor on a user's requirement.

The parameters have also be optimal for the patient's clinical status. The heart rate has here special importance: at the high cardiac rate the registered imaging data have also to be reconstructed very quickly (practically immediately). This is available only for the newest scanners and is applied for paediatric procedures. As usual, it does not exceed $65 \mathrm{bpm}$ but the required value depends on the scanner software. Lowering of the heart rate is achieved pharmacologically.

After completing acquisition of primary data elaboration on them begins, i.e. post-processing.

The possibilities of CT scanner are determined by the installed version of software which may be changed.

The principal algorithms for image post-processing are [14]:

- curved and multiplanar reformats (MPR),

- shaded-surface display (SSD),

- maximum intensity projection (MIP),

- volume rendering (VR).

The axial images produced by hardware (so-called row data) are essential and should be used but reformed images may improve lesion detection and classification (particularly coronal and oblique views). The axial images and MPRs are used for diagnosis and the SSD and MIPs are for display purposes.

The results of CTA are evaluated or consulted by a cardiologist.

\subsection{Dosimetric quantites}

Practical dosimetric quantities used for CT are a dose-length-product (DLP) and computedtomography-dose- index (CTDI). 
A dose-length-product (DLP) is directly measured by a dosemeter with a pencil chamber. Measured in units of $\mathrm{mGy} . \mathrm{cm}$, DLP reflects the integrated radiation dose (in a given position) for the total length of scanning.

The first estimation of a dose received by a patient during the CT procedure is the value of the $\mathrm{CTDI}_{\mathrm{vol}}$ displayed on the console monitor of the scanner. The CTDI ${ }_{\mathrm{vol}}$ represents an average dose over the scanned volume. The quantity is computed on the basis of exposure parameters (voltage, mAs per scan, pitch) according to the calibration stored in the computer memory of the scanner. The calibration is performed for the standard dosimetric cylindrical phantom from PMMA. By convention, a phantom $16 \mathrm{~cm}$ in diameter is used to model the head, and a $32 \mathrm{~cm}$ phantom is used to model the body (for adult patient). The displayed $\mathrm{CTDI}_{\mathrm{vol}}$ is used as a measure of radiation charge in the procedure.

$\mathrm{CTDI}_{\mathrm{vol}}$ can be computed on the basis of DLP measurements using a practical formula given below:

$\mathrm{CTDI}_{\mathrm{vol}}=\left(\mathrm{DLP}_{\mathrm{ave}} / \mathrm{L}\right)[\mathrm{mGy}]$

where $\operatorname{DLP}_{\mathrm{ave}}=(1 / 3) \mathrm{DLP}_{\mathrm{c}}+(2 / 3) \mathrm{DLP}_{\mathrm{p}}$, L- the accurate length of scan., i.e. the difference between start and end positions of the table.

$\mathrm{DLP}_{\mathrm{c}}$ and $\mathrm{DLP}_{\mathrm{p}}$ are measured in the centrum and at periphery of the adequate PMMA phantom (i.e. "HEAD" $\varnothing=16 \mathrm{~cm}$ or "BODY" $\varnothing=32 \mathrm{~cm}$ ).

The absorbed organ doses and then the effective dose received by a patient from a given CT procedure may be evaluated by the following approaches:

a. with calculation based on dosimetric measurements made in physical phantoms,

b. with Monte Carlo simulations using mathematical phantoms,

c. with $\mathrm{CTDI}_{\mathrm{vol}}$ values provided on the scanner console and a body region-specific conversion coefficient averaged for multiple scanners.

Dosimetric measurements are commonly made with termoluminescent dosimeters (lithium fluoride or calcium fluoride) or solid state detectors. The detectors are distributed inside the phantom which is exposed as a patient. The most popular are Rando Man or Rando Woman phantoms (representing adult humans) and the family of CIRS phantoms (representing children as well as adults).

Monte Carlo simulation assumes a mathematical phantom representing a patient from a given age group and model photon transitions through this phantom. The older solutions used the "family" of Cristy' phantoms for simulation, modelling organs as geometrical solids. The newer studies use voxel phantoms which are more anatomical being created on the basis of CT human images.

The last approach, i.e. taking some conversions coefficient from literature and multiplying by $\mathrm{CTDI}_{\mathrm{vol}}$ is the simplest, but under condition, that the both values are really adequate for the given scanner. 
Regardless of the way, the effective dose estimates the radiation charge for some "typical" (standard) patient, without taking into consideration the anatomy of a given human, and thus should be referred to the category of patients undergoing the given x-ray procedure.

\subsection{Doses to patients and radiation protection in CTA}

Computerized tomography is treated as a high-dose imaging technique although in the currently produced scanners a lot of mechanisms for dose reduction is implemented and the high doses can be avoided.

Because of exposure conditions in CTA (thin slices and low pitch) the doses to patients are relatively high, especially at 64-slice scanners. It was an inspiration to implement the methods of dose reduction without loss of image quality. (According to Sun et.al [1] 64-slice CT scanners are the most commonly used for coronary CT angiography, although 16-slice are still in use. The next place have dual-source CT scanners as dedicated to angiographic procedures.)

The mean effective doses in CTA for adult patients undergoing the procedure using 64-slice scanners are from $8 \mathrm{mSv}$ to $21.4 \mathrm{mSv}$ [6].

The doses can be reduced through lowering current-voltage parameters, implementation of x-rays intensity modulation (for three axis), shortening of scanned body length and ECG gating ( specially for cardiac procedures). ECG-controlled tube current modulation is very common approach utilized in CTA [1].

An example of the impact of different scan protocols on radiation dose and image quality is taken from [15]. The data concern the randomly selected adult patients examined for the evaluation of coronary artery disease. Two CT scanners were used: 16-slice and 64-slice.

\begin{tabular}{|c|c|c|c|c|c|c|}
\hline & \multicolumn{3}{|c|}{16 -slice CT } & \multicolumn{3}{|c|}{ 64-slice CT } \\
\hline & $120 \mathrm{kV}$ without & 120 kV & 100 kV & 120 kV without & 120 kV & 100 kV \\
\hline & EGC & with EGC & with EGC & EGC & with EGC & with EGC \\
\hline No. of patients & 30 & 50 & 50 & 50 & 50 & 30 \\
\hline Heart rate $[\mathrm{bpm}]$ & $61.3 \pm 11.3$ & $60.7 \pm 9.5$ & $57.8 \pm 5.3$ & $61.1 \pm 10.4$ & $57.5 \pm 7.2$ & $57.0 \pm 8.2$ \\
\hline $\begin{array}{l}\text { Tube current } \\
{[\mathrm{mA}]}\end{array}$ & $510.0 \pm 40.3$ & $304.5 \pm 42.3$ & $387.6 \pm 18.9$ & $870.0 \pm 55.6$ & $551.0 \pm 58.2$ & $537.8 \pm 50.7$ \\
\hline $\mathrm{CTDI}_{\mathrm{vol}}[\mathrm{Gy}]$ & $42.1 \pm 3.6$ & $25.2 \pm 2.9$ & $19.4 \pm 1.0$ & $58.8 \pm 6.5$ & $38.3 \pm 3.11$ & $22.0 \pm 1.8$ \\
\hline $\begin{array}{l}\text { Signal-to-noise } \\
\text { ratio }\end{array}$ & $11.1 \pm 3.9$ & $11.9 \pm 4.3$ & $11.9 \pm 3.7$ & $8.9 \pm 2.5$ & $9.2 \pm 2.8$ & $9.2 \pm 2.5$ \\
\hline $\begin{array}{l}\text { Effective dose } \\
\text { [mSv] }\end{array}$ & $10.6 \pm 1.2$ & $6.4 \pm 0.9$ & $5.0 \pm 0.3$ & $14.8 \pm 1.8$ & $9.4 \pm 1.0$ & $5.4 \pm 1.1$ \\
\hline
\end{tabular}

Table 3. Data for different protocols of CTA (from [15]) 
The special solution for dose reduction being dedicated to cardiac CT examinations is the change of image acquisition technique from RGH to PGA [8].

RGH $=$ Retrospectively Gated Helical: the patient and table move through the gantry at a steady speed. A low pitch (0.2 to 0.4 ) is needed to cover the entire cardiac volume, especially to compensate for any ectopic beats, which can result in misregistration and gaps in coverage. Thus, the same anatomy can be exposed to the x-ray beam many times (up to five) to ensure enough coverage, causing high absorbed doses.

PGA = Prospectively Gated Axial: the table is stationary during PGA image acquisition, then moves to the next location for another scan that is initiated by the next normal cardiac cycle.

The x-rays emission is then dynamically predicted on the basis of ECG signal.

Diagnostic value of images obtained for PGA protocol was found not lower than for RGH while the doses where substantially lower [8]. In the same study including 203 CTA exams (82 with routine RGH and 121 with the PGA) the doses were evaluated for protocol including scout images, low-dose calcium scoring scans, test-bolus scan and CT coronary angiogram. The exams were performed using 64-slice CT scanner (LightSpeed VCT XT, GE). The effective doses were as follows:

RGH: (8.7-23.2) mSv, mean: $18.4 \mathrm{mSv}$;

PGA: (0.75-6.67) mSv; mean: $2.84 \mathrm{mSv}$.

The similar dose values (16.3 $\mathrm{mSv}$ and $3.5 \mathrm{mSv}$ for RGH and PGA, respectively) are drawn as a conclusion from the papers published from 2007 to 2011 [1] and prospective ECG-triggering is recommended as the most commonly applied technique for dose reduction [16, 17].

Lowering of tube voltage is another technique frequently proposed for dose reduction. The results of multicenter, multivendor randomized trial using $80 \mathrm{kVp}$ was published by LaBounty et. al. [18]. The authors concluded that CTA using $80 \mathrm{kVp}$ instead of $100 \mathrm{kVp}$ was associated with a nearly $50 \%$ reduction in radiation dose with no significant difference in interpretability and noniferior image quality despite lower signal-to-noise and contrast-tonoise ratios.

\section{C-arm CT}

The newest technique for cardiac imaging is so-called C-arm CT (also Rotational Angio, dyna-CT). This is a special option of the new models of C-arm units introduced as a potential modality for intra-operative 3D imaging. This is possible for C-arm systems equipped with flat panel detectors and appropriate software for image reconstructions.

The C-arm is rotated over a wide range $\left(>180^{\circ}\right)$ with or without continuous contrast injection, acquiring multiple views of the cardiovascular structure with subsequent 3D reconstruction. This is advanced post processing giving images like 3D reconstructions from CT. For ECG-referenced cardiac imaging, identical alternating forward and backward rotations 
are triggered by the ECG signal to acquire projections covering the entire acquisition range at a similar cardiac phase $[19,20,21]$.

Because of the relatively slow rotation of the C-arm, the temporal resolution of these systems is significantly inferior to the new multislice CT scanners [19].

The acquisition of 3D data directly from the angiography system may facilitate co-registration of angiographic data with pre-procedurally acquired 3D CT images, with subsequent automatic registration to the 2D-fluoroscopy images obtained using the same system. The availability of real-time 3D anatomical information from the patient may offer advantages beyond those of pre-procedural images.

$\mathrm{C}$-arm CT as a technical solution joins some benefits of $\mathrm{CT}$ with $\mathrm{C}$-arm techniques, giving possibility to continue the diagnostic procedure (angiography) as a therapeutic one, and subsequently to provide CT-like images during an interventional procedure without moving the patient to a CT or MRI scanner.

The results of comparison of both CA modes (CCA and rotational angiography) performed for the same patients (over 200) revealed a hih degree of diagnostic agreement for 3 independent cardiologist and for each coronary segment [22]. Contrast medium volume during rotational CA and conventional CA amounted to $31.9 \pm 4.5 \mathrm{~mL}$ versus $52.2 \pm 8.0 \mathrm{~mL}$, and patient radiation exposure amounted to $5.0 \pm 2.6 \mathrm{~Gy} . \mathrm{cm}^{2}$ versus $11.5 \pm 5.5 \mathrm{~Gy} . \mathrm{cm}^{2}$, respectively.

\section{Author details}

Maria Anna Staniszewska

Dept. of Medical Imaging Techniques, Medical University, Lodz, Poland

\section{References}

[1] Sun Z, Ng K-H. Coronary CT angiography in coronary artery disease. WJC, 2011 September 26; 3(9): 303-310

[2] Advances in the Diagnosis of Coronary Atherosclerosis, Edited by Suna F.Kirac. InTech, 2011. Chapter 5: Coronary Angiography- Physical and Technical Aspects, by M.A.Staniszewska

[3] Vano E, Gonzales L, Guibelalde E, et al:Radiation exposure to medical staff in interventional and cardiac radiology. Br.J.Radio., 71,954-960,1998.

[4] International Commission on Radiological Protection, Publication 85, Avoidance of Radiation Injuries from Medical Interventional Procedures, 2001.

[5] http://www.cathlabdigest.com/articles/Biplane-Coronary-Angiography, by M.Kern 
[6] Einstein AJ, Moser KW, Thompson RC, Cerqueira MD, Hezlova MJ. Contemporary Reviews in Cardiovascular Medicine. Circulation, 2007; 116: 1290-1305

[7] Vano E, Arranz L, Sastre JM, et al: Dosimetric and radiation protection considerations based on some cases of patient skin injuries in interventional cardiology. Br.J.Radiol., 71, 510-516, 1998.

[8] Bor D, Olgar T, Toklu T, et al.: Patient doses and dosimetric evaluations in interventional cardiology. Physica Medica, 25,31-42, 2009.

[9] International Commission on Radiological Protection, Publication 75, General Principles of Monitoring for Radiation Protection of Workers, 1997.

[10] Padovani R: Optimisation of patient and staff exposure in interventional radiology. In: Radiation Protection in Medical Physics, edited by Y.Lemoigne and A.Caner, Springer in Cooperation with NATO Public Diplomacy Division, The Netherlands, 2011.

[11] Taylor A, Cerquiera M in Journal of the American College of Cardiology, October 2010: according to AuntMinnie.com (November 1,2010): New cardiac CT guidelines expand use for low-risk patients.

[12] Hamon M, Biondi-Zoccai GG, Malagutti P, Agostoni P, Morello R, Valgimigli M, Hamon M. Diagnostic performance of multi slice spiral computed tomography of coronary arteries as compared with conventional invasive coronary angiography. A meta-analysis. J Am Coll Cardiol 2006; 48: 1896-1910

[13] Wink O. Hecht HS, Ruijters D. Coronary computed tomographic angiography in the cardiac catheterization laboratory: current applications and future developments. Cardiol Clin 2009; 27: 513-529

[14] W.A.Kalender: Computed Tomography. Publicis MCD Verlag, Munich 2000

[15] Hausleiter J, Meyer T, Hademitzky M, Huber E, Zankl M, Martinoff S, Kastrati A, Schomig A. Radiation Dose Estimates From Cardiac Multislice Computed Tomography in Daily Practice. Circulation, 2006, March; 113:1305-1310

[16] Sabarudin A, Sun Z, Ng K-H. Radiation dose associated with coronary CT angiography and invasive coronary angiography: an experimental study of the effect of dose-saving strategies. Radiat Prot Dosimetry, 2012; 150(2): 180-187

[17] Hong YJ, Kim SJ, Lee SM, Min PK, Yoon YW, Lee BK, Kim TH. Low-dose coronary computed tomography angiography using prospective ECG-triggering compared to invasive coronary angiography. Int J Cardiovasc Imaging, 2011 Mar; 27(3): 425-431

[18] LaBounty TM, Leipsic J, Poulter R, Wood D, Johnson M, Srichai MB, Cury RC, Heibron B, Hague C, Lin FY, et al. Coronary CT angiography of patients with a normal body mass index using $80 \mathrm{kVp}$ versus $100 \mathrm{kVp}$ : a prospective, multicenter, multivendor randomized trial. Am J Radiol, 2011, Nov,; 197(5): W860-867 
[19] Schoenhagen P, Numburi U, Halliburton SS, Aulbach P, von Roden M, Desai MY, Rodriguez LL, Kapadia SR, Tuzcu EM, Lytle BW. Three-dimensional imaging in the context of minimally invasive and tanscatheter cardiovascular interventions using multi-detector computed tomography: from pre-operative planning to intra-operative guidance. European Heart Journal, 2010; 31: 2727-2741

[20] Neubauer AM, Garcia JA, Messenger JC, Hansis E, Kim MS, Klein AJ, Schoonenberg GA, Grass M, Carroll JD. Clinical feasibility of a fully automated 3D reconstructions of rotational coronary X-ray angiograms. Circ Cardiovasc Interv, 2010; 3: 71-79

[21] Tommassini G, Camerini A, Gatti A, Derelli G, Bruzzone A, Veccio G. Panoramic coronary angiography. J Am Coll Cardiol, 1998; 31: 871-877

[22] Empen K, Kuon E, Hummel A, Gebauer C, Dorr M, Konemann R, Hoffmann W, Staudt A, Weitmann K, Reffelmann T, Felix SB. Comparison of rotational with conventional coronary angiography. Am Heart J, 2010, Sept.; 160(3): 552-563 